\title{
Front acceleration by dynamic selection in Fisher population waves
}

\author{
O. Bénichou, ${ }^{1}$ V. Calvez, ${ }^{2}$ N. Meunier ${ }^{3}$ and R. Voituriez ${ }^{1}$ \\ ${ }^{1}$ Laboratoire de Physique Théorique de la Matière Condensée, \\ UMR 7600 CNRS /UPMC, 4 Place Jussieu, 75255 Paris Cedex \\ ${ }^{2}$ Unité de Mathématiques Pures et Appliquées, CNRS UMR 5669, E̛ équipe-projet INRIA NUMED, \\ École Normale Supérieure de Lyon, 46 allée d'Italie, F-69364 Lyon, France. \\ ${ }^{3}$ MAP5, CNRS UMR 8145, Université Paris Descartes, \\ 45 rue des Saints-Pères, 75270 Paris Cedex 06, France
}

(Dated: June 12, 2012)

\begin{abstract}
We introduce a minimal model of population range expansion in which the phenotypes of individuals present no selective advantage and differ only in their diffusion rate. We show that such neutral phenotypic variability can yield alone phenotype segregation at the front edge even in absence of genetic noise, and significantly impact the dynamical properties of the expansion wave. We present an exact asymptotic traveling wave solution and show analytically that phenotype segregation accelerates the front propagation. The results are compatible with field observations such as invasions of cane toads in Australia or bush crickets in Britain.
\end{abstract}

PACS numbers:

\section{INTRODUCTION}

The combination of random mutations and natural selection plays a key role in evolution. In the case of large well-mixed populations, it is found typically that mutations that eventually become dominant must present a selective advantage (i-e a larger effective growth rate) over the wild type [1]. More recently, it appeared that the case of expanding populations, which are a common phenomenon in biology, can lead to strikingly different behaviors. It was observed that in population range expansions even neutral mutations, i-e mutations that present no selective advantage, can prevail at least transiently due to large fluctuations at the edge of the population traveling front, and lead to spectacular gene segregation phenomena [2-4]. Such population waves are exemplified by the hypothesized migration of humans from Africa [5], or invasions of species such as cane toads in Australia [6] or bush crickets in Britain [7], and can now be observed at a smaller scale on the example of migrations of microorganisms in a Petri dish [3]. Following these observations, numerical models have been developed in the ecology community to analyze quantitatively range expansions [8-13]; these studies give further support to the mechanism of phenotype selection, which was dubbed spatial sorting in [14].

The standard mathematical models for population waves are generally based on the Fisher-Kolmogorov (FKPP) equation $[15,16]$, which is the simplest non linear equation that combines diffusion and growth. Beyond population genetics, this equation has been widely used for example in ecology, epidemiology [17] or chemistry [18]. Fisher waves are still actively investigated from the theoretical point of view and despite this effort, exact results, which mostly concern the propagation speed $[19,20]$, remain elusive especially in the presence of noise or in space dimensions larger than one.

In this letter, we introduce a minimal model of popu- lation dynamics based on a modified FKPP equation in which mutations are neutral and affect only the mobility properties of individuals. Our approach is inspired by the observations of range expansions of cane toads in Australia [6] or bush crickets in Britain [7], as well as several numerical studies[8-14] which highlighted the following features: (i) The leading part of the expanding front is mostly populated by the fastest phenotypes, that is longer-legged individuals in the case of cane toads [6] and longer-winged individuals in the case of bush crickets. (ii) The proportion of fast phenotypes is the highest in locations of new arrivals and then declines over time. (iii) The speed of the population wave increases with time. Our analysis is based on a modified FKPP equation with an additional mutation term that allows for variations of the diffusion rate of individuals, for which we present an exact asymptotic traveling wave solution and calculate analytically the front speed. We show that such phenotypic variability, when taken into account, can yield alone the segregation of the fastest phenotypes at the front edge in absence of genetic noise, and significantly accelerate the propagation speed. Importantly, the results reproduce qualitatively the observations (i)-(iii) above made on the basis of field experiments and numerical simulations. The novelty of our approach lies on the modified FKPP equation that we propose in Eq.(1), which enables an analytical derivation of the main properties of phenotypic segregation in range expansions. We analyze in this paper this equation in its minimal form, keeping in mind that it could be modified to account more faithfully for real systems. Given the wide spectrum of situations described by the FKPP equation, potential applications for example in the context of chemical kinetics are also expected. 


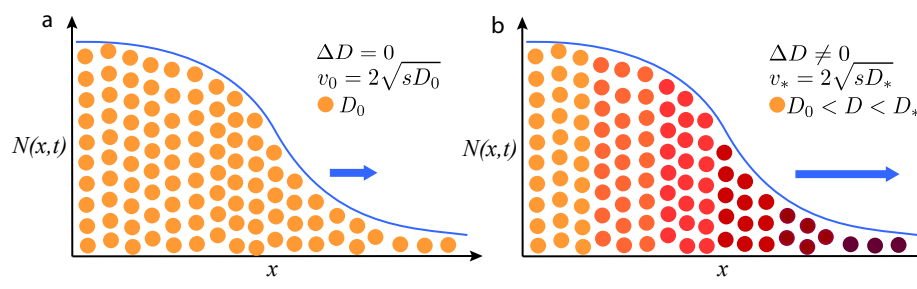

FIG. 1: Fisher population waves for (a) a homogeneous population with diffusion rate $D_{0}$ and for (b) a population with mutations that affect the diffusion rate so that $D \in\left[D_{0}-\Delta D / 2, D_{0}+\Delta D / 2\right]$. It is found in case $\mathbf{b}$ that a traveling wave solution exists and propagates faster than the classical velocity of a FKPP wave $v_{0}=2 \sqrt{s D_{0}}$ (case a). The leading edge of the front is mostly populated by fastest individuals (with diffusion rate $D_{*}>D_{0}$ ) that are selected dynamically, while the population behind the front is characterized by the steady state value $D_{0}$.

\section{MODEL}

We consider the following $1 d$ model (see Fig. 1). We assume that each individual of a growing population is characterized by a diffusion coefficient $D$, which is subject to mutations, and we denote by $n(D, x, t)$ the density of such individuals of phenotype $D$ at position $x$ and time $t$. We will also make use of the marginal density $N(x, t)=\int_{0}^{\infty} n(D, x, t) d D$ and the probability distribution of the trait $D$ at $(x, t)$ defined by $P_{x}(D, t) \equiv$ $n(D, x, t) / N(x, t)$. We assume that mutations, which only affect $D$, are neutral and do not affect the population growth rate $s$. Mutations are described phenomenologically by a density current $j[n(D, x, t)]$ in the phenotype space (an explicit example is given below). The dynamics is then given by the following modified FKPP equation :

$$
\partial_{t} n=D \partial_{x}^{2} n+\operatorname{sn}(1-N)-\partial_{D} j
$$

The diffusion term on the right-hand side accounts for the mobility of the individuals, which is effectively modelled by a diffusion process of coefficient $D$. The logistic term $\operatorname{sn}(1-N)$ accounts for the birth and death of individuals, where the growth rate $s$ is independent of the phenotype $D$. Note that here we omit number fluctuations of the birth and death process. Last, the divergence of the density current $-\partial_{D} j$ accounts for the dynamics of mutations.

To get insight into the effect of the mutation term, we first consider the case of a well-mixed population in a bounded range (for example $x \in[0,1]$ ). The stationary state (see also [21] for the analysis of a related problem) is then given by $N(x, t)=1$ for all $x$, with $P_{x}(D, t) \equiv P_{0}(D)$ independent of $x$ and defined by $j=0$. The function $P_{0}(D)$ therefore characterizes the steadystate distribution of the trait $D$ in a well-mixed population. We will consider the representative case of a distribution $P_{0}$ that is centered around a mean value $D_{0}$ with a typical width $\Delta D$. Without loss of generality, a simple choice for the density current is then

$$
j=-\alpha \partial_{D} n(x, D, t)
$$

where $\alpha$ is the mutation rate, together with reflecting boundary conditions $(j=0)$ at $D=D_{-} \equiv D_{0}-\Delta D / 2$ and $D=D_{+} \equiv D_{0}+\Delta D / 2$. With this choice of $j$, the distribution $P_{0}$ is then uniform over the range $\left[D_{-}, D_{+}\right]$ and the different phenotypes have no selective advantage. Another example of interest is given by

$$
j=-\alpha\left[\beta\left(D-D_{0}\right) n(x, D, t)+\partial_{D} n(x, D, t)\right],
$$

where $\alpha$ is the mutation rate and the extra term $\beta$ accounts here for a selective advantage of phenotype $D_{0}$. This choice, together with the zero flux condition at $D=$ $D_{-}$and $D=D_{+}$, yields $P_{0}(D) \propto \exp \left[-\beta\left(D-D_{0}\right)^{2} / 2\right]$ : at stationary state in a bounded range, the trait $D_{0}$ is now favored, as is the case in many real situations. Alternative choices are possible to account more faithfully for field experiments, and would lead qualitatively to similar results. We will focus below on the minimal choice of Eq.(2), which captures the main features of the model and keep calculations analytically tractable.

We now turn to the case of a range expansion and assume that at $t=0$ the population is seeded with the uniform distribution $P_{0}$ in a bounded interval of the $x$ axis. We show that despite the fact that mutations are neutral, a traveling wave solution characterized by a non homogeneous distribution of $D$, which we calculate in the regime $u \equiv x-v_{*} t \gg 1$, emerges. We determine the velocity $v_{*}$ and show that it is larger than the velocity $v_{0}=2 \sqrt{s D_{0}}$ expected from the classical FKPP model with an homogeneous population of diffusion rate $D_{0}$.

Let us introduce $u \equiv x-v t$ where $v$ is to be determined, and look for a traveling front so that $N(u \rightarrow-\infty)=1$ and $N(u \rightarrow+\infty)=0$. Notations are kept unchanged in the co-moving frame of velocity $v$ and all functions of $x$ and $t$ are assumed to depend on $u$ only. Eq. (1) then rewrites

$$
0=v \partial_{u} n+D \partial_{u}^{2} n+\operatorname{sn}(1-N)+\alpha \partial_{D}^{2} n .
$$

The key point of the following analysis is that one can show self-consistently that in the limit $u \rightarrow \infty$, one has $P_{x}(D, t) \equiv P_{u}(D) \rightarrow P_{*}(D)$, where $P_{*}(D)$ is independent of $u$. The dependences on $u$ and $D$ in $n(D, u)$ are therefore factorized at the leading edge of the front and one can write in this limit: $n(D, u) \simeq P_{*}(D) N(u)$.

This factorized form of the solution can be shown to hold in a mathematically rigorous framework which will be presented elsewhere. We give here the main ingredients of derivation. In the regime $u \rightarrow \infty, n$ is small and Eq.(4) can be linearized, which suggests the following ansatz

$$
n(D, u)=P_{*}(D) \exp (-\gamma u)
$$

with $\gamma>0$. Eq.(4) then rewrites

$$
0=\left(-\gamma v+D \gamma^{2}+s\right) P_{*}(D)+\alpha \partial_{D}^{2} P_{*}(D),
$$



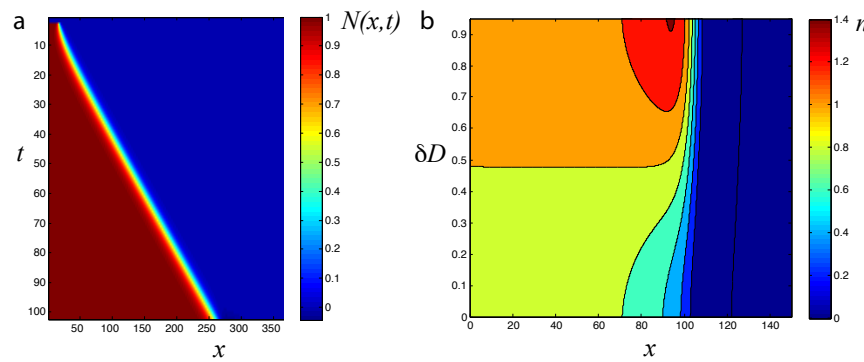

FIG. 2: Traveling wave solution of Eq.(1) obtained numerically for $D_{0}=1.5, \Delta D=1, s=1$ and $\alpha=0.01$. a Kymograph showing the propagation of the marginal density $N(x, t)$. b Distribution of phenotypes in the traveling wave at a given time $t$ in the stationary state (the front is approximately at $x=90$ ). A larger heterogeneity corresponding to phenotype segregation is found at the leading edge of the front.

where it should be noted that $P_{*}(D)$ depends on $\gamma$. The speed $v$ of a front of prescribed profile characterized by $\gamma$ is then obtained by integration over $D$ and follows:

$$
v=D_{*} \gamma+s / \gamma
$$

where $D_{*}$, which depends on $\gamma$, is determined below. Eq. (6), completed by $\left.\partial_{D} P_{*}\right|_{D=D_{-}}=\left.\partial_{D} P_{*}\right|_{D=D_{+}}=0$ for the choice (2) of the density current $j$, explicitly defines the function $P_{*}$ and shows that it is independent of $u$ in the limit $u \rightarrow \infty$, therefore proving that the factorized form of Eq. (5) holds true. We stress that this argument holds for any density current $j$, which would only modify the ordinary differential equation (6) defining $P_{*}$. In the explicit example of Eq. (2), the existence of a non zero solution for Eq. (6) implies that :

$$
\begin{aligned}
\operatorname{Ai}\left[1, \mu\left(D_{*}-D_{-}\right)\right] \operatorname{Bi}\left[1, \mu\left(D_{*}-D_{+}\right)\right] \\
-\operatorname{Bi}\left[1, \mu\left(D_{*}-D_{-}\right)\right] \operatorname{Ai}\left[1, \mu\left(D_{*}-D_{+}\right)\right]=0
\end{aligned}
$$

where $\mu=\left(\gamma^{2} / \alpha\right)^{1 / 3}$ and $\mathrm{Ai}$ and Bi denote Airy functions. This last equation, which can not be solved analytically to the best of our knowledge, implicitly defines $D_{*}$ as a function of $\gamma$, and completes the definition of $v$ in Eq.(7). The distribution $P_{*}$ is then given by

$$
Z P_{*}=\operatorname{Ai}\left[\mu\left(D_{*}-D\right)\right]-\frac{\operatorname{Ai}\left[1, \mu\left(D_{*}-D_{-}\right)\right]}{\operatorname{Bi}\left[1, \mu\left(D_{*}-D_{-}\right)\right]} \operatorname{Bi}\left[\mu\left(D_{*}-D\right)\right],
$$

where $Z$ is a normalization constant that ensures that $\int_{D_{-}}^{D_{+}} P_{*}(D) d D=1$. This result, together with the decoupling form of Eq.(5) explicitly characterizes the asymptotics of a front of prescribed profile characterized by $\gamma$. In practice, it can be showed following classical arguments [18] that in the case of a localized initial condition in space the slowest solution is selected. The critical velocity $v_{*}$ (and the corresponding $\gamma_{*}$ ) is therefore obtained as the minimum of the function $v(\gamma)$ defined by Eqs. (7) and (8), and the density for $u$ large follows

$$
n(D, u) \propto P_{*}(D) e^{-\gamma_{*} u} .
$$

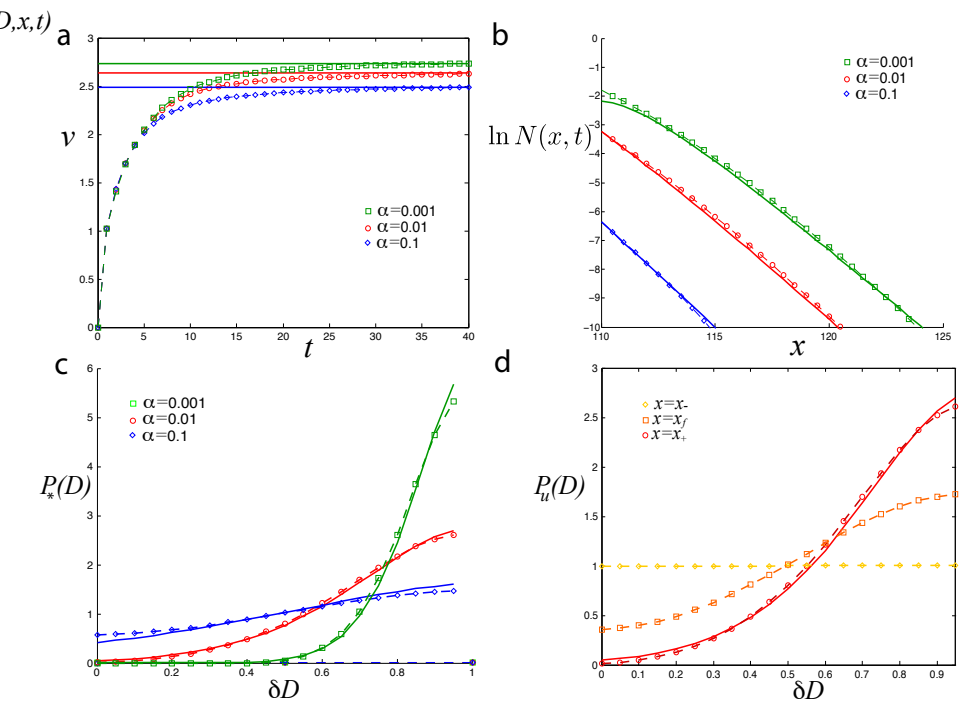

FIG. 3: Numerical simulations of Eq.(1) for $D_{0}=1.5$, $\Delta D=1$ and $s=1$. a. Front velocity as a function of time for different values of the mutation rate $\alpha$. The front is accelerated until it reaches the exact predicted velocity in the stationary state (plain line). b. Shape of the front (log scale) at the leading edge in the stationary state. The theoretical prediction (plain lines) is compared to numerical simulations (symbols and dashed lines) for different values of the mutation rate $\alpha$. c. Probability distribution of the trait $D$ at the leading edge in the stationary state, where $\delta D \equiv D-D_{-}$. The theoretical prediction of Eq. (10) (plain lines) is compared to numerical simulations (symbols and dashed lines). d. Probability distribution of the trait $D$ in the stationary state at different positions $x$, where $\delta D \equiv D-D_{-}$. Here $\alpha=0.01$, $x_{f}$ denotes the position of the front and $x_{-}=x_{f}-50$ and $x_{+}=x_{f}+10$. The theoretical prediction $P_{*}$ (plain line) holds at the leading edge $x_{+}$while the distribution is homogeneous at $x_{-}$, which is the steady state value $P_{0}$ for the choice of Eq. (2).

While in practice $v_{*}$ can be determined by a numerical analysis of Eqs.(7) and (8), a useful and very accurate approximate of $v_{*}$, which is in fact an exact upper bound, denoted $v_{*}^{u}$, can be obtained by assuming $D_{*}$ constant in Eq.(7). Under this hypothesis the minimization of $v$ is realized for $\gamma=\gamma_{*}^{u} \equiv \sqrt{s / D_{*}}$ and yields $v_{*}^{u}=2 \sqrt{s D_{*}}$, where $D_{*}$ is determined by Eq. (8) taken at $\gamma=\gamma_{*}^{u}$.

These asymptotic results have been checked numerically by solving Eq. (1) using a classical Euler numerical scheme, the diffusion part being considered implicitly and the reaction part explicitly, and show that the upper bound $v_{*}^{u}$ provides a very accurate estimate of the exact value $v_{*}$. Figure 2 shows an example of the obtained population wave which reaches a stationary state of constant velocity after a transient acceleration regime (Fig. 2a). Figure 2b indicates that the population is the most heterogeneous at the edge of the front where the segregation of the fastest phenotypes is observed, while variations of the trait $D$ decrease behind the front. More quantitatively, we find that after the transient accelera- 
tion regime the wave advances at the predicted velocity $v_{*}$ (see Fig. 3a). Numerical results clearly validate the asymptotic result of Eq. (10) as seen in Fig. 3b,c, which show that both the dependences on $u$ and $D$ of the density $n(D, u)$ are captured by our analysis. Remarkably, the approximate value $v_{*}^{u}$ is numerically extremely close to the exact result $v^{*}$ for all parameters analyzed. Last, Fig. 3d confirms that the segregation of phenotypes with larger diffusion rate $D$ is more important at the leading edge of the front, where it is characterized by the predicted distribution $P_{*}(D)$, while the distribution $P_{u}(D)$ reaches its steady state value $P_{0}(D)$ (which is uniform with the choice of Eq.(2)) at positions $x$ far behind the front.

\section{DISCUSSION}

Several comments are in order. First, the asymptotic solution of Eq. (10) and the determination of the front velocity $v_{*}$ are exact. Similar results, and in particular the exact asymptotic decoupling of the $u$ and $D$ dependences can be obtained along the same lines for other choices of the density current $j$ defined in Eq. (2). In particular, the important case where a trait $D_{0}$ presents a selective advantage, as modeled by the current defined in Eq. (3), can be analyzed in the same way and leads to qualitatively similar results. Second, one finds that for $s / \alpha \ll 1, D_{*} \rightarrow D_{0}$ and $P_{u}(D) \simeq P_{0}(D)=1 / \Delta D$ for all $u$. As expected, when mutations are extremely fast the population is locally well-mixed everywhere with diffusion rate $D_{0}$ and the dynamics follows the usual FKPP equation with a front speed $v_{0}=2 \sqrt{s D_{0}}$. In the more realistic case of slow mutations, $s / \alpha$ is large and the dynamics selects faster individuals at the lead- ing edge so that $P_{*}(D)$ favors larger values of $D$. In the extreme case of very slow mutations $(s / \alpha \rightarrow \infty)$ one has $D_{*} \rightarrow D_{+}$and $P_{*}(D) \simeq \delta\left(D-D_{+}\right)$. The front can then be significantly faster than the expected velocity $v_{0}$ for a well-mixed population of diffusion rate $D_{0}$ since $v_{*} \rightarrow 2 \sqrt{s\left(D_{0}+\Delta D / 2\right)}>v_{0}$. Third, it should be emphasized that this segregation of fastest phenotypes, which leads to an acceleration of the wave propagation, is purely dynamical. It is obtained in the low noise limit, and therefore strikingly differs from the mechanism of segregation of neutral mutations studied in [2-4]. Last, and importantly, our results qualitatively reproduce the observations (i)-(iii) stated in introduction. Indeed, we found that: (i) fastest phenotypes are segregated at the leading edge of the front, as shown in Figs. 2b, 3c. (ii) The proportion of fast phenotypes is higher at the edge and decreases behind the front as shown in Figs. 2b, 3d. (iii) The speed of advance of the population wave increases with time, as seen in Fig. 2a, and is larger than the expected propagation speed for a well-mixed population.

To conclude, we have proposed a minimal model of population range expansion in which mutations are neutral and affect only the mobility of individuals. We have shown that such neutral phenotypic variability can yield alone phenotype segregation at the front edge in absence of genetic noise, and significantly impact the dynamical properties of the expansion wave. An exact traveling wave solution can be obtained asymptotically, and shows that phenotype segregation accelerates the front propagation. The results are compatible with available data of invasions of cane toads in Australia or bush crickets in Britain. Applications beyond the field of population dynamics could be expected.
[1] J. F. Crow and M. Kimura, An Introduction to Population Genetics Theory (Blackburn Press, Caldwell NJ, 2009).

[2] C. A. Edmonds, A. S. Lillie, and L. L. Cavalli-Sforza, Proc Natl Acad Sci U S A 101, 975 (2004).

[3] O. Hallatschek, P. Hersen, S. Ramanathan, and D. R. Nelson, Proc Natl Acad Sci U S A 104, 19926 (2007).

[4] K. S. Korolev, M. Avlund, O. Hallatschek, and D. R. Nelson, Rev Mod Phys 82, 1691 (2010).

[5] N. A. Rosenberg, J. K. Pritchard, J. L. Weber, H. M. Cann, K. K. Kidd, L. A. Zhivotovsky, and M. W. Feldman, Science 298, 2381 (2002).

[6] B. L. Phillips, G. P. Brown, J. K. Webb, and R. Shine, Nature 439, 803 (2006).

[7] C. D. Thomas, E. J. Bodsworth, R. J. Wilson, A. D. Simmons, Z. G. Davies, M. Musche, and L. Conradt, Nature 411, 577 (2001).

[8] S. Klopfstein, M. Currat, and L. Excoffier, Mol Biol Evol 23, 482 (2006).

[9] C. L. Hughes, C. Dytham, and J. K. Hill, Ecological Entomology 32, 437 (2007).
[10] J. M. J. Travis, T. Münkemüller, O. J. Burton, A. Best, C. Dytham, and K. Johst, Mol Biol Evol 24, 2334 (2007).

[11] L. Excoffier and N. Ray, Trends Ecol Evol 23, 347 (2008).

[12] J. M. J. Travis, T. Münkemüller, and O. J. Burton, J Evol Biol 23, 2656 (2010).

[13] B. L. Phillips, G. P. Brown, J. M. J. Travis, and R. Shine, Am Nat 172 Suppl 1, S34 (2008).

[14] R. Shine, G. P. Brown, and B. L. Phillips, Proc Natl Acad Sci U S A 108, 5708 (2011).

[15] R. Fisher, Ann. Eugenetics 7, 353 (1937).

[16] A. Kolmogorov, N. Petrovsky, and N. Piscounov, Moscow Univ. Math. Bull. p. 1 (1937).

[17] J. Murray, Mathematical Biology (Springer, New York, 2003).

[18] W. Van Saarloos, Physics Reports 386, 29 (2003).

[19] E. Brunet and B. Derrida, Physical Review E 56, 2597 (1997).

[20] O. Hallatschek and K. S. Korolev, Phys Rev Lett 103, 108103 (2009).

[21] J. Dockery, V. Hutson, K. Mischaikow, and M. Pernarowski, Journal of Mathematical Biology 
37, 61 (1998).

\section{Acknowledgments}

N.M. wishes to thank C. Mouhot for having brought his attention on this subject and for helpful discussions. 